
Romenigue Igor Melo Araujo Fernandes

\title{
Obtenção das Forças de Usinagem Utilizando o Modelo de Jhonson-Cook
}

\author{
Monografia submetida ao curso de gra- \\ duação em Engenharia Aeroespacial da \\ Universidade de Brasília, como requisito par- \\ cial para obtenção do Título de Bacharel em \\ Engenharia Aeroespacial.
}

Universidade de Brasília - UnB

Faculdade UnB Gama - FGA

Orientador: Prof. Dr. Rhander Viana

Coorientador: Prof. Dr. Lucival Malcher

Brasília, DF

2019 
Romenigue Igor Melo Araujo Fernandes

Obtenção das Forças de Usinagem Utilizando o Modelo de Jhonson-Cook/

Romenigue Igor Melo Araujo Fernandes. - Brasília, DF, 2019-

44 p. : il. (algumas color.) ; $30 \mathrm{~cm}$.

Orientador: Prof. Dr. Rhander Viana

Trabalho de Conclusão de Curso - Universidade de Brasília - UnB

Faculdade UnB Gama - FGA , 2019.

1. Simulação Numérica. 2. Usinagem. I. Prof. Dr. Rhander Viana. II. Universidade de Brasília. III. Faculdade UnB Gama. IV. Obtenção das Forças de Usinagem Utilizando o Modelo de Jhonson-Cook 


\title{
Obtenção das Forças de Usinagem Utilizando o Modelo de Jhonson-Cook
}

\author{
Monografia submetida ao curso de gra- \\ duação em Engenharia Aeroespacial da \\ Universidade de Brasília, como requisito par- \\ cial para obtenção do Título de Bacharel em \\ Engenharia Aeroespacial.
}

Trabalho aprovado. Brasília, DF, 13 de Dezembro de 2019:

Prof. Dr. Rhander Viana

Orientador

Me. Danilo Santos Oliveira

Convidado 1

Profa. Dra. Maria Del Pillar Fidalgo

Falla

Convidado 2

Brasília, DF

2019 

Dedico este trabalho aos meus pais, Maria de Fátima e Carlos da Silva, por terem contribuído intensamente na minha educação. 



\section{Agradecimentos}

Agradeço primeiramente a minha mãe, Maria de Fátima, e ao meu Pai, Carlos da Silva, pelo suporte oferecido durante essa jornada. Por terem acreditado e apoiado cada decisão pessoal e profissional que tomei, mesmo quando o vento não era favorável. Agradeço também a minha irmã, Rejane Cristina, e ao meu irmão, Robson Henrique, pelo interesse em todos passos da minha graduação. Com todas as forças que regem o universo, digo-lhes que os amo.

Agradeço as minhas sobrinhas, Débora Cristina, Beatriz Natália, Natália Maria, Milena e Manuela. Vocês me motivam a continuar e a superar cada desafio. Sonho em ajudar cada uma de vocês. Com todo amor do mundo, obrigado por existirem.

Agradeço também ao meu querido amigo Raifran Lima por ter passado todo esse tempo ao meu lado. Fico feliz por ter dividido momentos de alegria e tristeza com você. Agradeço por todos os conselhos dados e por toda paciência oferecida. Sou grato por ter me ajudado nos momentos mais difíceis da minha vida. Você merece o mundo.

Por fim, agradeço o meu orientador Rhander Viana por ter me ajudado de todas as formas a conquistar este título. 
[...] Eu sou dono e senhor do meu destino;

Eu sou comandante de minha alma.

William E Henley 


\section{Resumo}

Simular numericamente um processo de usinagem é uma forma avançada de entender o comportamento das forças de corte envolvidas no processo de fabricação sem a necessidade de um experimento físico. O modelo de Jhonson - Cook foi proposto para definir o comportamento termo-elástico-plástico da peça bem como o ponto crítico de tensão onde ocorre a formação do cavaco no aço AISI 1045. Um modelo 3D foi modelado usando o Método dos Elementos Finitos no software ABAQUS para simular o processo de usinagem. O resultados obtidos na simulação foram satisfatórios e se manteve com uma diferença de $20 \%$ dos valores obtidos no dinamômetro Klister no experimento físico.

Palavras-chaves: Elementos Finitos, Forças de Corte, Usinagem, Simulação Computacional, Jhonson-Cook Model, ABAQUS. 


\section{Abstract}

Simulate a machining process is an important and advanced way to understand the behavior of forces involved in this process without a financial large cost. In this paper, The Johnson-Cook model was used to define thermo-elastic-plastic of workpiece as well as the damage criteria to reach the chip separation during the machining process of AISI 1045. An 3D geometry was modelled using Finite Element Method based on software ABAQUS to simulate a cutting process. The results obtained in this simulation was satisfactory being the difference around of 20

Key-words: Finite Element Method (FEM), Computational Simulation, Cutting Forces, Jhonson-Cook Model, Machining Process, ABAQUS. 


\section{Lista de ilustrações}

Figura 1 - Processos de Fabricação (Machado and Silva, 2004). . . . . . . . . . . . 22

Figura 2 - Direção dos movimento de corte no torneamento (Machado and Silva, 2004). . . . . . . . . . . . . . . . . . . 23

Figura 3 - Direção dos movimentos de corte no fresamento (Machado and Silva, 2004). . . . . . . . . . . . . . . . . . . . 24

Figura 4 - Direção dos movimentos de corte na furação (Machado and Silva, 2004). 24

Figura 5 - Zona de cisalhamento primária e secundária (Nascimento and Coelho, 2011). . . . . . . . . . . . . . . . . . 26

Figura 6 - Zona de cisalhamento primária e secundária (Diniz et al., 2001). . . . . 27

Figura 7 - Corte ortogonal: (a) vista tridimensional e (b) vista bidimensional do corte ortogonal (Groover, 2007) . . . . . . . . . . . . . . . 28

Figura 8 - Diagrama de forças (Groover, 2007). . . . . . . . . . . . 28

Figura 9 - Malha de Elementos Finitos. . . . . . . . . . . . . . . 30

Figura 10 - Geometrias de Elementos Finitos. . . . . . . . . . . . . . . . . 30

Figura 11 - Dinamometro Klister 9257B. . . . . . . . . . . . . . . . . . . . . 34

Figura 12 - Dinamometro Klister 9257B. . . . . . . . . . . . . . . . . . 35

Figura 13 - Modelagem 3D da peça e ferramenta. . . . . . . . . . . . . . . 36

Figura 14 - Simulação de usinagem 3D . . . . . . . . . . . . . . . . . . . . . . 37

Figura 15 - Formação do Cavaco. . . . . . . . . . . . . . . . . . . . . . . 37

Figura 16 - Análise das forças de corte no aço 1045. . . . . . . . . . . . . . . . 38 


\section{Lista de tabelas}

Tabela 1 - Propriedades Mecânicas do Tungstênio . . . . . . . . . . . . . . . 33

Tabela 2 - Propriedades Mecânicas do Aço 1045 . . . . . . . . . . . . . . 33

Tabela 3 - Constantes do modelho de falha proposto para Johnson-Cook para o

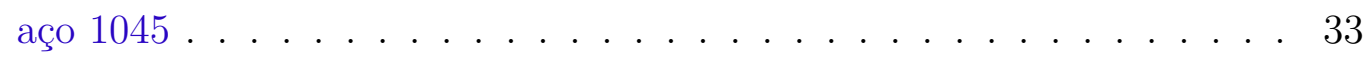

Tabela 4 - Parâmetros de corte . . . . . . . . . . . . . . 34

Tabela 5 - Inclinação da Ferramenta . . . . . . . . . . . . . . . . 34 


\section{Lista de abreviaturas e siglas}

FEM Finite Element Methods

MEF Método dos Elementos Finitos

3D Três Dimensões

2D Duas Dimensões

ALE Arbritary Lagrangian-Eulerian 



\section{Lista de símbolos}

$\begin{array}{ll}\Gamma & \text { Letra grega Gama } \\ \Lambda & \text { Lambda } \\ \zeta & \text { Letra grega minúscula zeta } \\ \epsilon & \text { Pertence }\end{array}$




\section{Sumário}

INTRODUÇÃO $\ldots \ldots \ldots \ldots \ldots \ldots \ldots \ldots$

1.1

OBJETIVOS

MATERIAIS E MÉTODOS . . . . . . . . . . . . . 33

Referências . . . . . . . . . . . . . . 43 


\section{INTRODUÇÃO}

Citar algum componente que não passou pelo processo de usinagem é uma tarefa difícil. Sem o processo de fabricação, aviões, trens e veículos certamente não existiriam. A concepção de produtos industriais e domésticos não seria viável sem um torno mecânico (Walker, 1989). Tais máquinas promovem agilidade e possibilidade de fabricar peças de geometrias complexas com acabamento satisfatório. A usinagem é um processo de fabricação caracterizado pela remoção de material de uma determinada matéria prima para dar forma a uma peça. O cavaco está diretamente relacionado com as propriedades do material que compõe a matéria prima, da ferramenta utilizada e dos parâmetros de corte inserido no torno. Durante o processo de usinagem, o material removido recebe o nome de cavaco. A formação do cavaco envolve diversas teorias de mecânica dos sólidos, materiais e transferência de calor. Segundo Diniz et al. (2001), força de corte é a ação da ferramenta em contato com a peça. Geralmente, métodos de mensurar força de corte pode ser dividido em duas categorias: força direta e força indireta (Scippa, 2015). Vários fatores podem influenciar a força de corte durante o processo de fabricação, como o material e velocidade de corte (Ferraresi, 1970). Estudar as forças de usinagem é inevitável para entender o seu comportamento de seus componentes. Tendo esse entendimento fica possível estimar a força requerida em um processo de corte, evitar o desgaste prematuro da ferramenta, obter uma ótima usinibilidade e colaborar com projetos e conceitos de ferramentas e máquinas (Machado and Silva, 2004).

A demanda global por produtividade e qualidade de produto culminou no avanço de pesquisa nos processos de fabricação. A evolução histórica na obtenção de resultados para definir parametros de usinagem aconteceu pelos métodos analiticos, experimentais e numéricos (Ehmann, 1997). O método analítico tenta estabelecer matematicamente relações entre força de corte e diversos aspectos mecânicos como atrito, geometria e comportamento mecânico dos materiais. O método empírico consiste em coletar dados obtidos pelo operador da máquina de torno, ou seja, compara o resultado final de uma determinada peça que passou pelo processo de usinagem (Gonzalo, 2008). A maneira mais comum de medir força de corte é através de um dinamômetro acoplado no torno. Geralmente os dinamômetros são compostos de sensores pizoelétricos que são montados entre duas placas (Albrecht et al., 2005).

O método numérico consiste na solução de equações através de ferramentas e teorias de cálculo numérico afim de se obter resultados refinados. Soluções computacionais são amplamentes usadas para resolver equações de alta complexidade de forma rápida e precisa. O Método dos Elementos Finitos (FEM) é uma ferramenta matemática ro- 
busta, confiável e amplamente usada por softwares de simulação para resolver problemas de engenharia. O FEM surgiu com a promessa de contribuir em aplicações em diversas áreas da mecânica relacionadas a engenharia Aeroespacial e Civil. Uma das mais importantes aplicações são em problemas combinados, tal como interações fluidodinâmicas, termomecânica, termoquímica, termo-química-mecânica, biomecânica, biomédica, ferroeletricidade e eletromagnetismo (Harish, 2019). O método consiste na discretização de uma estrutura específica, em outras palavras significa difivir a estrutura em diversas partes menores. Essas partes menores são chamadas de elementos. Cada elemento representa geralmente uma equação diferencial simples cuja solução pode ser obtida ou aproximada. O softwares ABAQUS é um ferramenta computacional que utiliza o FEM para resolver problemas de engenharia. O software segue três passos para encontrar uma solução de um problema: pre-processamento, solução e pós-processamento. No pré-processamento, a geometria, propriedades do material, condições de contorno, forças e tipo de análise é definido. O pré-processamento é o principal e mais importante passo para se obter resultados satisfatórios na simulação. Na etapa de solução ocorre todo os cálculos necessários para a resolução da matrix de equações diferenciais. O último passo é o pós-processamento, ou seja, nessa etapa se extrai valores, imagens e gráficos para análise dos resultados da simulação. Com o crescimento exponencial da utilização de computadores de alta performance e a precisão de softwares de engenharia atuais, metodos computacionais são cada vez mais adotados na indústria. Tal decisão de mercado culmina na redução de custo na pesquisa e na melhoria da produtividade.

\subsection{OBJETIVOS}

\subsubsection{Objetivo Geral}

O objetivo do presente trabalho é analisar dados experimentais e numéricos de forças de corte usando o método Jhonson-Cook para descrever as propriedades do materials e Arbitrary Lagrangian-Eulerian (ALE) como tipo de análise explícita para alcançar resultados que podem reduzir o tempo de usinagem, aumentar a vida útil de ferramentas e melhoria no processo de fabricação.

\subsubsection{Objetivos específicos}

Realizar uma revisão bibliográfica sobre o processo de usinagem, simulação explícita, equações de Jhonson-Cook e sobre o Método dos Elementos Finitos (FEM). 


\subsection{METODOLOGIA}

A metodologia aplicada divide-se nos seguintes passos:

- Utilizar o dinamômetro Klister modo 9257B com três canais para obtenção de dados analíticos.

- Utilizar o Método de Elementos Finitos (FEM) para obter a solução numérica.

- Uso do software ABAQUS versão 14.1 para obtenção de dados numéricos.

- Usar o aço AISI 1045 como material dos experimentos analíticos e numéricos. 



\section{Revisão Bibliográfica}

\subsection{Usinagem}

Até meados do século XVIII, o principal material utilizado para peças, em engenharia, era a madeira, salvo raras exceções, a qual era usinada com ferramentas de aço-carbono. Com a Revolução Industrial, novos e mais resistentes materiais apareceram, impulsionando o desenvolvimento dos aços-liga como ferramentas de corte. Mais tarde, a utilização da água e do vapor como fontes de energia impulsionaram a indústria metal-mecânica, já no final do século XVIII e início do século XIX, propiciando assim o aparecimento de máquinas-ferramentas responsáveis pela fabricação de outras variedades de máquinas e instrumentos em substituição ao trabalho humano em diversas atividades (Machado et al., 2015).

Entende-se como usinagem o processo de fabricação que consiste na retirada de material de uma determinada matéria prima a fim de se obter uma peça com acabamento, dimensão e geometria projetada. A usinagem é reconhecidamente o processo de fabricação mais popular do mundo, transformando em cavacos algo em torno de $10 \%$ de toda a produção de metais, e empregando dezenas de milhões de pessoas em todo o mundo (Trent and Wright, 2000).

O processo de usinagem tradicional consiste em torneamento, furação, alargamento, moldagem, plaina e brochamento, bem como o processo de abrasivos, tais como retificação, usinagem ultrassônica, batedura e afiação. Processos avançados utilizam eletricidade e produtos químicos para a remoção de material, além de jatos abrasivos, jatos de água e corte a laser (Kalpakjian et al., 2011). A Figura 1 mostra a classificação dos processos de fabricação. 


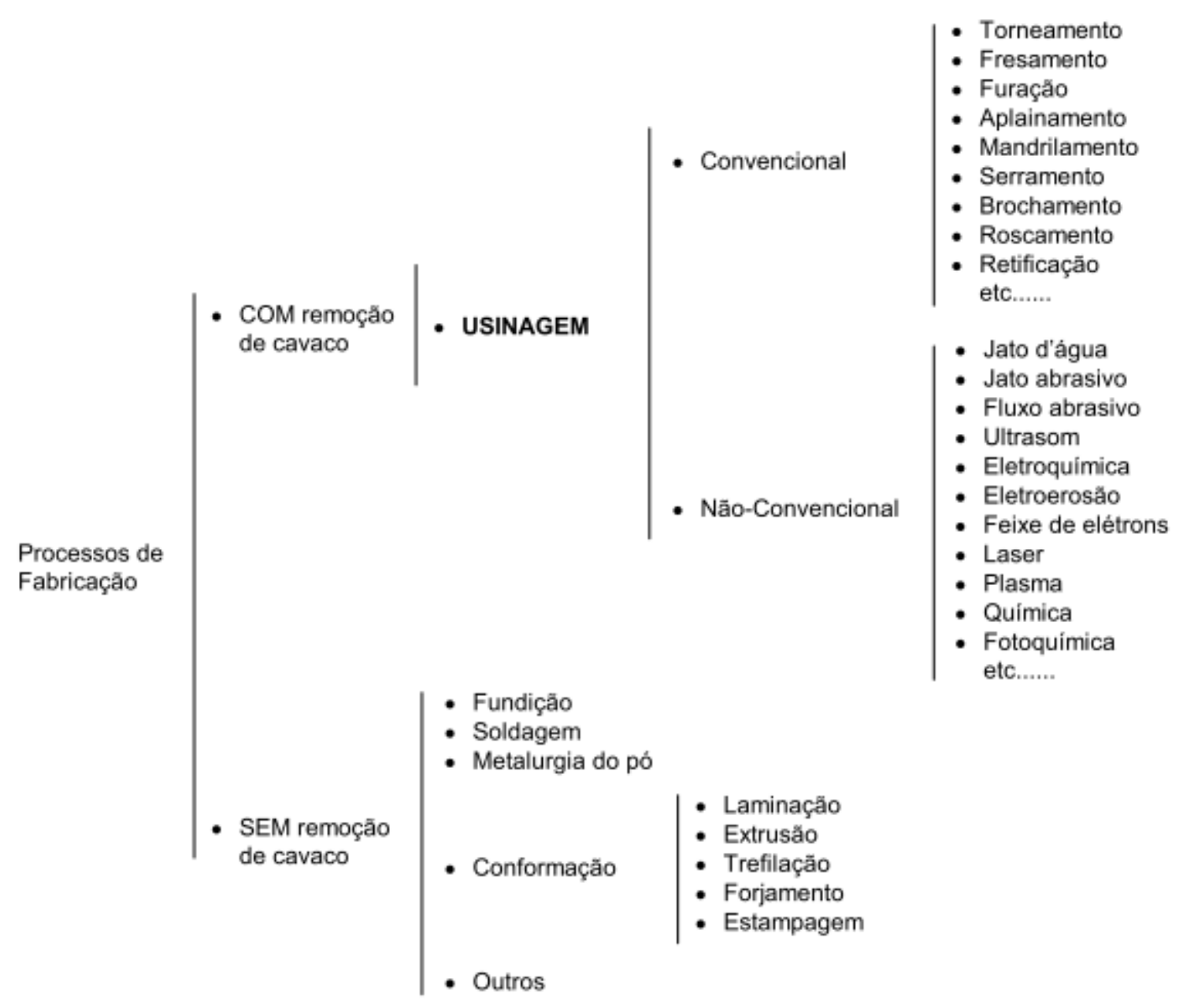

Figura 1 - Processos de Fabricação (Machado and Silva, 2004).

\subsubsection{Movimentos na Usinagem}

Os movimentos na usinagem proporcionam a ocorrência do processo de usinagem. O movimento é a interação entre ferramenta e peça. Por convenção, os movimentos são sempre descritos tendo como referencial a peça parada e a ferramenta realizando o movimento. Divide-se o movimento em ativos e passivos. Os movimentos classificados como ativos são aqueles que promovem a remoção de material. São eles:

- Movimento de corte: é o movimento principal que propicia o corte do material gerando o cavaco. O movimento é rotativo e realizado pela peça. Sem o movimento de avanço, o resultado será uma única remoção de cavaco durante uma revolução da ferramenta.

- Movimento de avanço: é o movimento que desloca a ferramenta ao longo da superfície da peça retirando material produzindo cavaco. 
- Movimento efetivo de corte: é a resultante dos movimentos de corte e de avanço realizados ao mesmo tempo.

Os demais movimentos são passivos, mesmo sendo fundamentais, não promovem a remoção de material.

- Movimento de ajuste: é o movimento entre a ferramenta e peça em direção ao interior da peça. Esse movimento pré-determina a espessura da camada de material a ser removida.

- Movimento de correção: é o movimento entre a ferramenta e peça que visa atenuar alterações de posicionamento resultantes, por exemplo, de desgaste da ferramenta, varições térmicas, deformações plásticas dentre outras.

- Movimento de aproximação: é movimento de aproximação da ferramentar à peça. Ocorre antes de iniciar o processo de usinagem.

- Movimento de recuo: é o movimento de afastamento da ferramenta após a usinagem.

As Fig 2, Fig 3 e Fig 4 mostram os movimentos de usinagem no torneamento, fresamento e furação.

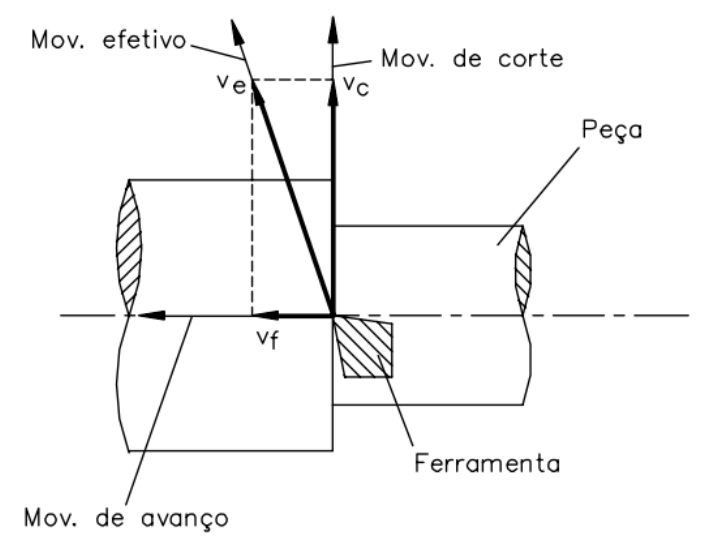

Figura 2 - Direção dos movimento de corte no torneamento (Machado and Silva, 2004).

\subsubsection{Velocidades}

Velocidade de corte $V_{c}$ é a velocidade instantânea do ponto de referência da aresta cortante da ferramenta, segundo a direção e o sentido do corte. Para processos com movimentos de rotação, a velocidade de corte é calculada pela Eq. (2.1) (Machado and Silva, 2004).

$$
V_{c}=\frac{\pi \cdot d \cdot v}{1000} \quad[\mathrm{~m} / \mathrm{min}]
$$




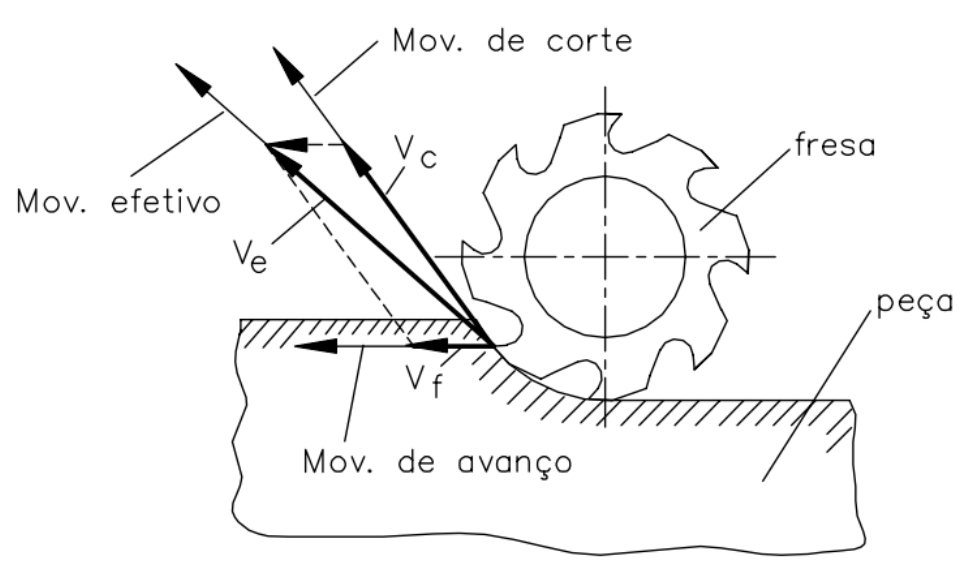

Figura 3 - Direção dos movimentos de corte no fresamento (Machado and Silva, 2004).

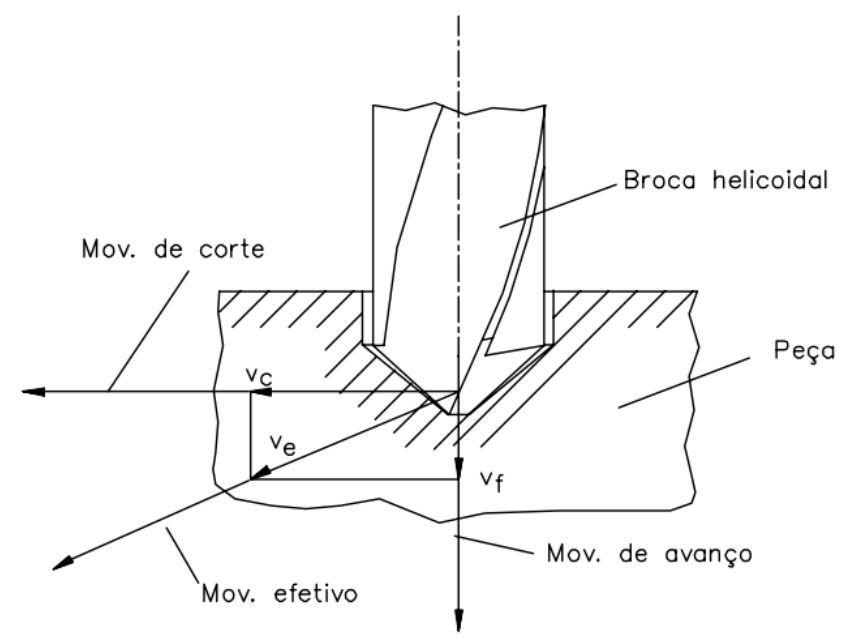

Figura 4 - Direção dos movimentos de corte na furação (Machado and Silva, 2004).

Onde:

- $\mathrm{d}=$ diâmetro da peça diâmetro da peça ou da ferramenta em $\mathrm{mm}$

- $\mathrm{n}$ = número de rotações por minuto (rpm)

A velocidade de avanço $V_{f}$ é a velocidade instantânea do ponto de referência da aresta cortante da ferramenta, segundo a direção e sentido de avanço. É dada pela Eq. $(2.2)$

$$
V_{f}=f \cdot n \quad[\mathrm{~mm} / \mathrm{min}]
$$

onde $\mathbf{f}$ é o avanço em mm/volta e $\mathbf{n}$ o número de rotações por minuto. 
A soma vetorial das duas velocidades descritas acima resulta na velocidade efetiva de corte $V_{e}$ como se segue na Eq. (2.3).

$$
\vec{V}_{e}=\vec{V}_{c}+\vec{V}_{f} \quad[\mathrm{~m} / \mathrm{min}]
$$

\subsubsection{Formação do Cavaco}

Durante o processo de corte, um das partes mais importantes é o comportamento tribológico na interação ferramenta-cavaco e seus efeitos nas variáveis de usinagem (Wan et al., 2018). Existem diversos estudos a respeito da formação do cavaco, pois a teoria da plasticidade não descreve com exatidão deformações em altíssimas velocidades seguida de ruptura. Está linha de pesquisa não está no escopo do presente trabalho, portanto, no será dado um entendimento geral sobre as fases de formação do cavaco e suas definições. Segundo Machado and Silva (2004), este processo pode ser dividido em quatro partes:

- Recalque inicial: devido à penetração da cunha cortante no material da peça, uma pequena porção deste (ainda unido à peça) é pressionada contra a superfície de saída da ferramenta.

- Deformação e ruptura: o material pressionado sofre, de início, uma deformação elástica, e, em seguida, uma deformação plástica, que aumenta progressivamente até o estado de tensões provocar a ruptura. Essa ruptura se dá, na maior parte das vezes, por cisalhamento, embora exista um estado de tensões que combina tensões de compressão/tração e cisalhamento e leve o material à ruptura, segundo algum critério próprio de sua natureza frágil ou dúctil. Após a ruptura, há a formação de uma trinca que se propaga seguindo também um critério de propagação de trincas próprio de cada material. Como a formação de cavacos ocorre dinamicamente, há planos instantâneos de ruptura e de propagação de trincas que definirão uma certa região entra a peça e o cavaco, chamada "zona primária de cisalhamento". Para facilitar o tratamento matemático dado à formação do cavaco, modelos simples assumem essa região como sendo apenas um plano matemático, ou seja, "o plano de cisalhamento", no qual se concentra, preferencialmente, a ruptura. Zona de cisalhamento primário é mostrada na Fig. 5

- Deslizamento de lamelas: continuando a penetração da ferramenta na peça, haverá uma ruptura parcial, ou completa, na região de cisalhamento, dependendo da extensão da propagação da trinca. As propriedades do material e as condições de avanço e de velocidade de corte irão determinar quanto o seguimento do material rompido permanecerá unido ao cavaco recém-formado, dando origem a cavacos contínuos ou descontínuos, conforma a extensão e a resistência da união entre as lamelas de material rompido. 
- Saída do cavaco: devido ao movimento relativo entre a ferramenta e a peça, inicia-se um escorregamento da porção de material deformada e rompida - o cavaco - sobre a superfície de saída da ferramenta. Enquanto tal evento ocorre, uma nova lamela do material (imediatamente adjacente à porção anterior) está se formando e passando pelos mesmos processos. Essa nova porção de material também escorregará sobre a superfície de saída da ferramenta, repetindo mais uma vez o fenômeno.

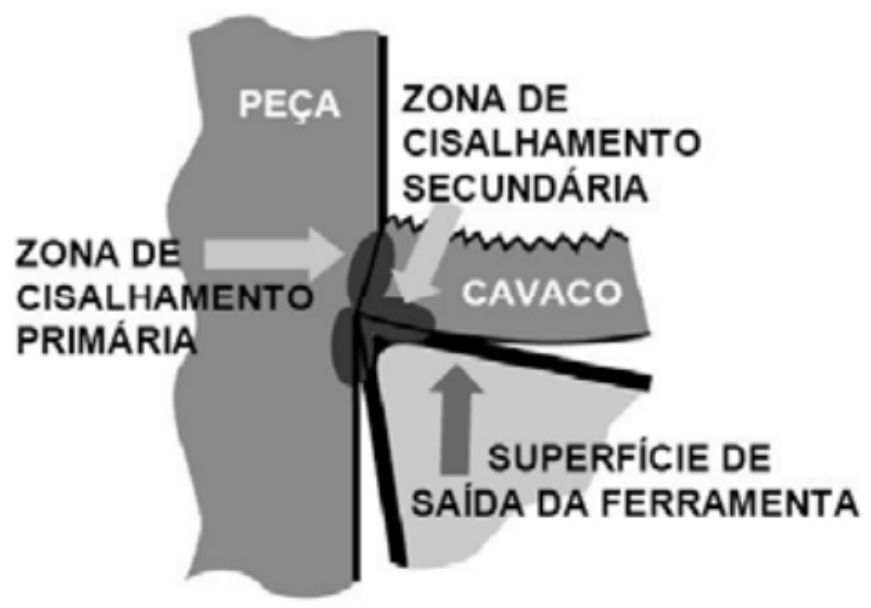

Figura 5 - Zona de cisalhamento primária e secundária (Nascimento and Coelho, 2011).

\subsubsection{Forças de Corte}

As forças de usinagem são consideradas como uma ação da peça sobre a ferramenta. A força total resultante que atua sobre a cunha cortante durante a usinagem é chamada força de usinagem $F_{0}$. A princípio, nem a direção, nem o sentido da força de usinagem são conhecidos, tornando-se impossível medi-la e conhecer melhor as influências de diversos parâmetros no seu valor Diniz et al. (2001). As forças de corte são dividas em três componentes:

- Força principal de corte $F_{0}$ : é a projeção da força de usinagem sobre o plano de trabalho, na direção de corte, dada pela velocidade de corte.

- Força de avanço $F_{f}$ : é a projeção da força de usinagem sobre o plano de trabalho, na direção de avanço, dada pela velocidade de avanço.

- Força passiva ou força de profundidade $F_{p}$ : é a projeção da força de usinagem perpendicular ao plano de trabalho.

Além das forças apresentadas, há outras três componentes importantes. 
- Força ativa $F_{T R}$ : é a projeção da força de usinagem sobre o plano de trabalho.

- Força de compressão $F_{n}$ : é a projeção da força de usinagem sobre uma direção perpendicular à direção de avanço, situada no plano de trabalho.

- Força de apoio $F_{a p}$ : é a projeção da força de usinagem sobre uma direção perpendicular à direção de avanço, situada no plano de trabalho.

A decomposição da força de usinagem resulta em três componentes principais:

$$
F_{U}=\sqrt{F_{a p}^{2}+F_{f}^{2}+F_{p}^{2}}
$$

A figura 6 apresenta os vetores de força no plano de trabalho em um fresamento.

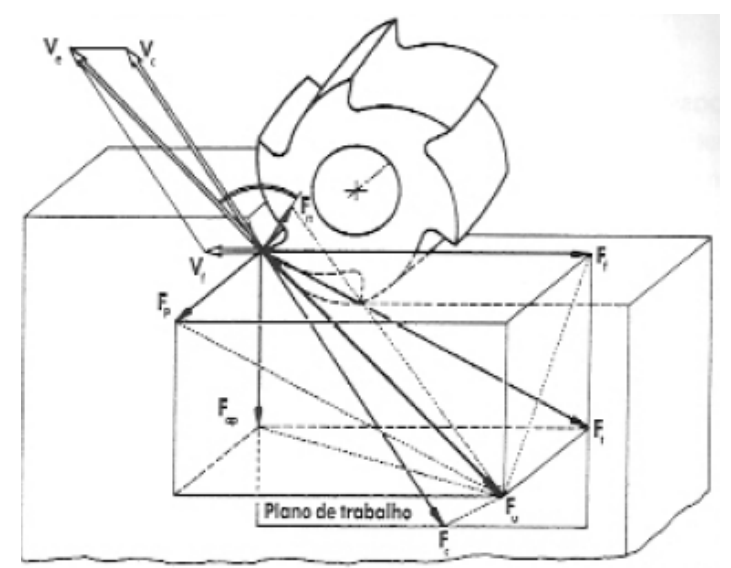

Figura 6 - Zona de cisalhamento primária e secundária (Diniz et al., 2001).

\subsubsection{Corte Ortogonal}

Por definição, corte ortogonal utiliza uma ferramenta no formato de cunha no qual o sua ponta de corte é perpendicular a direção da velocidade de corte Groover (2007).

A geometria do corte ortogonal permite estabelecer uma importante relação entre a espessura do cavado, angulo de entrada e o plano de cisalhamento. Tendo $l_{s}$ como comprimento do plano de cisalhamento e substituindo: $t_{0}=l_{s} \sin \phi$ e $t_{c}=l_{s} \cos (\phi-\alpha)$. Então:

$$
r=\frac{l_{s} \sin \phi}{l_{s} \cos (\phi-\alpha)}=\frac{\sin \phi}{\cos (\phi-\alpha)}
$$

Rearranjando a equação, podemos obter o ângulo do plano de cisalhamento $\phi$ dessa forma:

$$
\tan \phi=\frac{r \cos \alpha}{1-r \sin \alpha}
$$


A Fig. 7 abaixo evidencia o corte ortogonal:

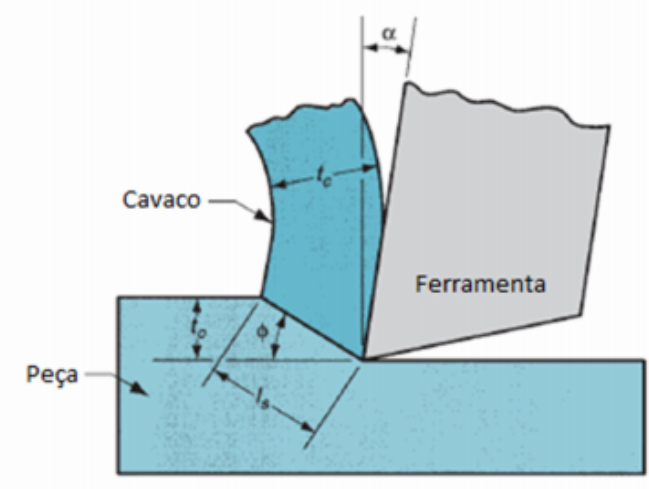

Figura 7 - Corte ortogonal: (a) vista tridimensional e (b) vista bidimensional do corte ortogonal (Groover, 2007).

O modelo ortogonal de corte é uma forma de simplificar o entendimento da atuação das forças de corte. Neste modelo, todos os componentes atuam em um único plano.

A decomposição da força de usinagem $F_{U}$ nas diversas direções obedece a um teorema da geometria que permite representar todos os componentes em um círculo, onde $F_{U}$ é o seu diâmetro Machado and Silva (2004). Essa representação é chamada de Círculo de Merchant.

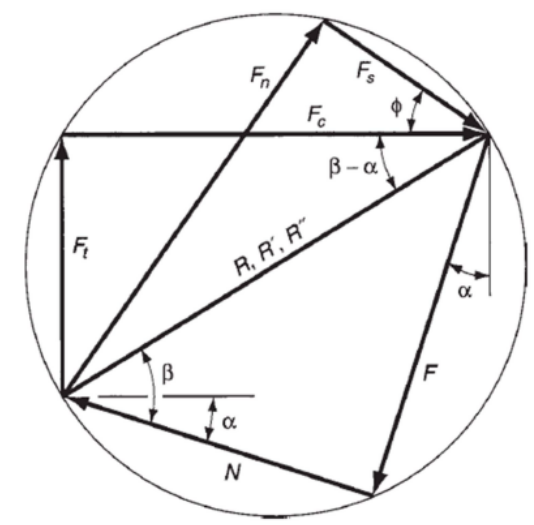

Figura 8 - Diagrama de forças (Groover, 2007).

As forças representadas na figura 8 se relacionam da seguinte forma:

$$
\begin{array}{ll}
F=F_{c} \operatorname{sen} \alpha+F_{t} \cos \alpha & {[N]} \\
N=F_{c} \cos \alpha-F_{t} \operatorname{sen} \alpha & {[N]} \\
F_{s}=F_{c} \cos \phi-F_{t} \sin \phi & {[N]}
\end{array}
$$




$$
F_{n}=F_{c} \sin \phi+F_{t} \cos \phi \quad[N]
$$

Tendo a tensão de cisalhamento e a área do plano de cisalhamento:

$$
\begin{gathered}
\tau=\frac{F_{f}}{A_{s}} \\
A_{s}=\frac{t_{0} w}{\operatorname{sen} \phi}
\end{gathered}
$$

Com as equações de força e da equação (2.12) é possível reescrever a equação para a tensão de cisalhamento da seguinte forma:

$$
\tau=\frac{F_{c} \cos \phi-F_{t} \operatorname{sen} \phi}{\frac{t_{0} w}{\operatorname{sen} \phi}}
$$

Merchant utilizou o princípio da Mínima Energia para determinar o ângulo $\phi$. Segundo este princípio, de todos os ângulos de cisalhamento possíveis, haverá um que predominará: aquele que minimiza a energia necessária para que o corte ocorra (de Andrade Dimas, 2018).

Derivando a equação (2.13) e igualando a zero, obtêm-se a Equação de Merchant:

$$
\phi=\frac{\pi}{4}-\frac{\beta}{2}+\frac{\alpha}{2}
$$

\subsection{MEF - Método dos Elementos Finitos}

O Método dos Elementos Finitos (MEF) consiste em um método numérico aproximado para análise de diversos fenômenos físicos que ocorrem em meios contínuos, e que são descritos através de equações diferenciais parciais, com determinadas condições de contorno (Problemas de Valor de Contorno), e possivelmente com condições iniciais (para problemas variáveis no tempo). O MEF é bastante genérico, e pode ser aplicado na solução de inúmeros problemas da engenharia (Souza, 2003).

A ideia principal do Método dos Elementos Finitos consiste em se dividir o domínio (meio contínuo) do problema em sub-regiões de geometria simples (formato triangular, quadrilateral, cúbico, etc.), conforme ilustra esquematicamente a Figura 9. Esta ideia é bastante utilizada na engenharia, onde usualmente tenta-se resolver um problema complexo, subdividindo-o em uma série de problemas mais simples. Logo, trata-se de um procedimento intuitivo para os engenheiros (Souza, 2003). 


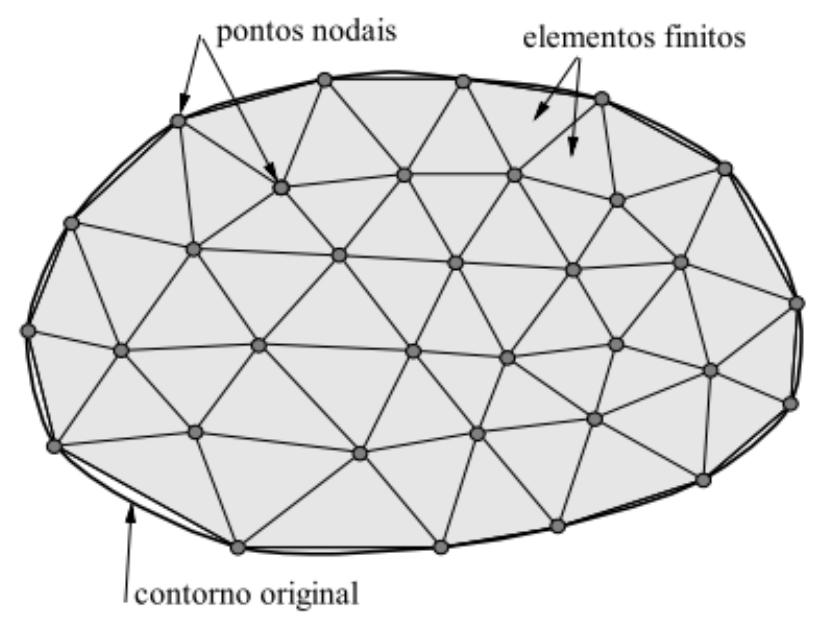

Figura 9 - Malha de Elementos Finitos.

Esta ideia é bastante utilizada na engenharia, onde usualmente tenta-se resolver um problema complexo, subdividindo-o em uma série de problemas mais simples. Logo, trata-se de um procedimento intuitivo para os engenheiros (Souza, 2003).

Os elementos finitos utilizados na discretização (subdivisão) do domínio do problema são conectados entre si através de determinados pontos, denominados nós ou pontos nodais, conforme indica a Figura 9. Ao conjunto de elementos finitos e pontos nodais, dáse, usualmente o nome de malha de elementos finitos (Souza, 2003).

Diversos tipos de elementos finitos já foram desenvolvidos. Estes apresentam formas geométricas diversas (por exemplo, triangular, quadrilateral, cúbico, etc) em função do tipo e da dimensão do problema (se uni, bi, ou tridimensional). A Figura 10 apresenta a geometria de vários tipos de elementos finitos (Souza, 2003).

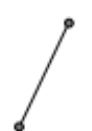

Elemento de barra com dois nós

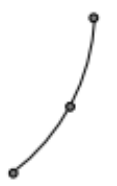

Elemento de barra com três nós

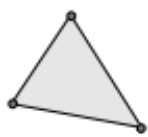

Elemento triangular com três nós

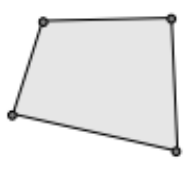

Elemento quadrilateral com quatro nós

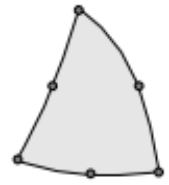

Elemento triangular com seis nós

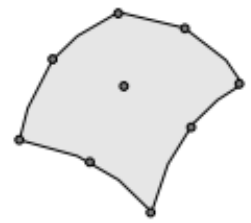

Elemento quadrilateral com nove nós

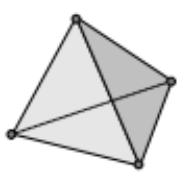

Elemento tetraédrico com quatro nós

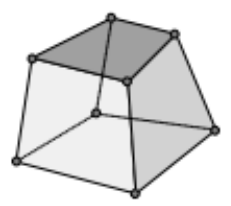

Elemento hexaédrico com oito nós

Figura 10 - Geometrias de Elementos Finitos. 
A precisão do método depende da quantidade de nós e elementos, e do tamanho e tipo dos elementos presentes na malha. Um dos aspectos mais importantes do MEF diz respeito a sua convergência. Embora trata-se de um método aproximado, pode-se demonstrar que em uma malha consistente, a medida que o tamanho dos elementos finitos tende a zero, e consequentemente, a quantidade de nós tende a infinito, a solução obtida converge para a solução exata do problema. Ou seja, quanto menor for o tamanho e maior for o número de elementos em uma determinada malha, mais precisos serão os resultados da análise (Souza, 2003).

\subsection{Modelo de Johnson-Cook}

Johnson e Cook propôs o modelo em 1983. Esse é um modelo no qual é amplamente utilizado em códigos computacionais para análise estática e dinâmica, como a usinagem em metais, crash tests e etc (Dehkharghani, 2016).

O modelo de fratura de Johnson-Cook foi o primeiro a definir o fluxo de tensão de deformações plasticas, taxa de deformação e temperatura. Esse modelo assume que o dano acumulado em um dado elemento do material durante a deformação plástica acelera rapidamente quando a falha atinge um valor crítico (Banerjee et al., 2015).

A equação define o fluxo de tensão proposto por Johnson-Cook:

$$
\sigma=\left[A+B(\varepsilon)^{n}\right]\left[1+C \ln \left(\frac{\dot{\varepsilon}}{\dot{\varepsilon_{0}}}\right)\right]\left[1-\left.\left(\frac{T-T_{0}}{T_{m e l t}-T_{0}}\right)\right|^{m}\right]
$$

Onde $\sigma$ é o fluxo de tensão e $\varepsilon$, $\dot{\varepsilon}$ e $\dot{\varepsilon_{0}}$ significa a tensão plástica efetiva, taxa de deformação efetiva e taxa de deformação de referência. Os parâmetros $T, T_{\text {melt }}$ e $T_{0}$ são temperatura absoluta, temperatura de fusão e temperatura de referência. As constantes dos materiais são $A, B, C, m$ e $n$. $A$ é a tensão de ruptura do material sob as condições de referência, $B$ é a constante de dureza, $n$ é o coeficiente de dureza, $C$ o coeficiente de resistência a taxa de deformação e $m$ o coeficiente térmico.

\subsubsection{Modelo de Johnson-Cook para Fratura}

$\mathrm{Na}$ análise de elementos finitos, dois critérios são frequentemente utilizados para separar o cavaco da superfície usinada: um critério geométrico e a critério de equivalência de deformação plástica. A deformação plástica equivalente são populares e efetivas na modelagem de separação do cavaco do material usinado (Mabrouki and Rigal, 2006). Johnson e Cook propuseram que a fratura depende da taxa de tensão triaxial, taxa de deformação e temperatura (Banerjee et al., 2015). O modelo de fratura proposto por 
Johnson e Cook é dado pela equação abaixo:

$$
\varepsilon_{f}=\left[D_{1}+D_{2} \exp \left(D_{3}\left(\frac{\sigma_{m}}{\sigma_{e q}}\right)\right)\right]\left[1+D_{4} \ln \left(\frac{\dot{\varepsilon}}{\dot{\varepsilon_{0}}}\right)\right]\left[1+D_{5}\left(\frac{T-T_{0}}{T_{m e l t}-T_{0}}\right)\right]
$$

Onde $D_{1}$ a $D_{5}$ são contantes do modelo de falha, $\sigma_{m}$ é a tensão principal e $\sigma_{e q}$ é a tensão equivalente. 


\section{Materiais e Métodos}

Neste capítulo será apresentado a metodologia e os equipamentos utilizados para obtenção dos resultados.

\subsection{Ferramenta e Peça}

Para modelar a ferramenta e a peça no ABAQUS ou em qualquer outro software de simulação é necessário conhecer as propriedades mecânicas dos materiais que irão compor cada parte. O valor das propriedades mecânicas e físicas irão influenciar diretamente na simulação. Em estudos anteriores, o inserto de tungstênio, metal duro, foi estabelecido como um material ideal para a ferramente que irá usinar o aço 1045 (Kalpakjian et al., 2011). O aço 1045 foi adotado por apresentar excelente usinabilidade. As propriedades dos materiais estão presentes na Tab 3.1 e 3.1

Tabela 1 - Propriedades Mecânicas do Tungstênio

\begin{tabular}{|c|c|c|c|c|}
\hline Densidade & Poisson & Módulo de Elasticidade & Condutividade Térmica & Calor Específico \\
\hline $11.900 \frac{\mathrm{kg}}{\mathrm{m}^{3}}$ & 0.22 & $534 \mathrm{GPa}$ & $50 \mathrm{Wm}^{-1} \mathrm{C}^{-1}$ & $400 \frac{\mathrm{J}}{\mathrm{kg}} \mathrm{C}$ \\
\hline
\end{tabular}

Tabela 2 - Propriedades Mecânicas do Aço 1045

\begin{tabular}{|c|c|c|c|c|c|}
\hline Densidade & Módulo de Young & Módulo de Poisson & Módulo de Cisalhamento & Condutividade Térmica & Calor Específico \\
\hline $7850 \frac{\mathrm{kg}}{\mathrm{m}^{3}}$ & $200 \mathrm{GPa}$ & 0.29 & $80 \mathrm{GPa}$ & $52 \mathrm{Wm} \mathrm{m}^{-1} \mathrm{C}$ & $486 \frac{\mathrm{J}}{\mathrm{kg}} \mathrm{C}$ \\
\hline
\end{tabular}

Os critérios de falha de Johnson-Cook adotado para o aço 1045 são mostrados na Tab 3.1.

Tabela 3 - Constantes do modelho de falha proposto para Johnson-Cook para o aço 1045

\begin{tabular}{|l|l|}
\hline Parâmetros de Falha & Aço AISI 1045 \\
\hline$D_{1}$ & 0.06 \\
\hline$D_{2}$ & 3.31 \\
\hline$D_{3}$ & -1.96 \\
\hline$D_{4}$ & 0.0018 \\
\hline$D_{5}$ & 0.58 \\
\hline
\end{tabular}

As constantes $A, B, C$, nem do aço 1045 são dados na tabela abaixo:

\begin{tabular}{|l|l|c|c|l|}
\hline A (MPa) & B (MPa) & $\mathbf{C}$ & $\mathbf{n}$ & $\mathbf{m}$ \\
\hline 553.1 & 600.8 & 0.0134 & 0.234 & 1 \\
\hline
\end{tabular}




\subsection{Metodologia}

Para o desenvolvimento do experimento com objetivo de obter as forças de corte, foi adotado alguns procedimentos de usinagem. O experimento ocorreu com a profundidade de corte constante igual a $1 \mathrm{~mm}$ e avanço constante igual a $0.094 \frac{\mathrm{mm}}{\mathrm{rot}}$. Todo processo de remover material foi realizado sem fluido de corte. O inserto de metal duro sem revestimento tem especificação CNMG120408H13A fabricado pela Sandvik. A ferramenta foi modelada no software com a mesma geometria especificada abaixo. As condições de corte são mostrada na Tab 3.2. A ferramenta foi montada com ângulo de corte $X_{r}$, ângulo de saída $Y_{0}$ e inclinação $\lambda_{s}$ dado na Tab 3.2.

Tabela 4 - Parâmetros de corte

\begin{tabular}{|l|c|c|c|}
\hline & Velocidade de Corte $\mathbf{( m / m i n})$ & Avanço $\mathbf{~ m m / r o t )}$ & Profundidade de corte $\mathbf{( m m})$ \\
\hline $\mathbf{1}$ & 51.8 & 0.094 & 1 \\
\hline $\mathbf{2}$ & 82.8 & 0.094 & 1 \\
\hline $\mathbf{2}$ & 207.2 & 0.094 & 1 \\
\hline
\end{tabular}

Tabela 5 - Inclinação da Ferramenta

\begin{tabular}{|l|l|l|}
\hline$X_{r}$ & $Y_{0}$ & $\lambda_{s}$ \\
\hline 95 & -6 & -6 \\
\hline
\end{tabular}

\subsection{Dinamômetro e Torno}

A dinamômetro Klister modelo 9257B com três canais foi usado para medir a força de corte durante a usinagem Fig. 11. O dinamômetro é amplamente usado em pesquisas e de desenvolvimento por conta de suas diversas funcionalidades, sensores robustos ao ponto de coletar dados com alta precisão. O módulo do dinamômetro é responsável por processar informações obtidas durante o processo de usinagem em tempo real.

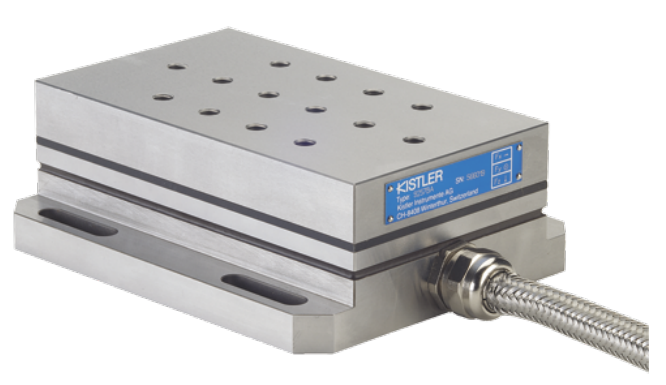

Figura 11 - Dinamometro Klister 9257B. 
O dinamômetro estacionário foi montado na torre de ferramenta do torno Nardini Nodus 220 com 5 cv de acordo com o manual do fabricante. Dessa forma, o dinamômetro ficou entre a ferramenta e a torre do torno, conforme mostrado na Fig 12.

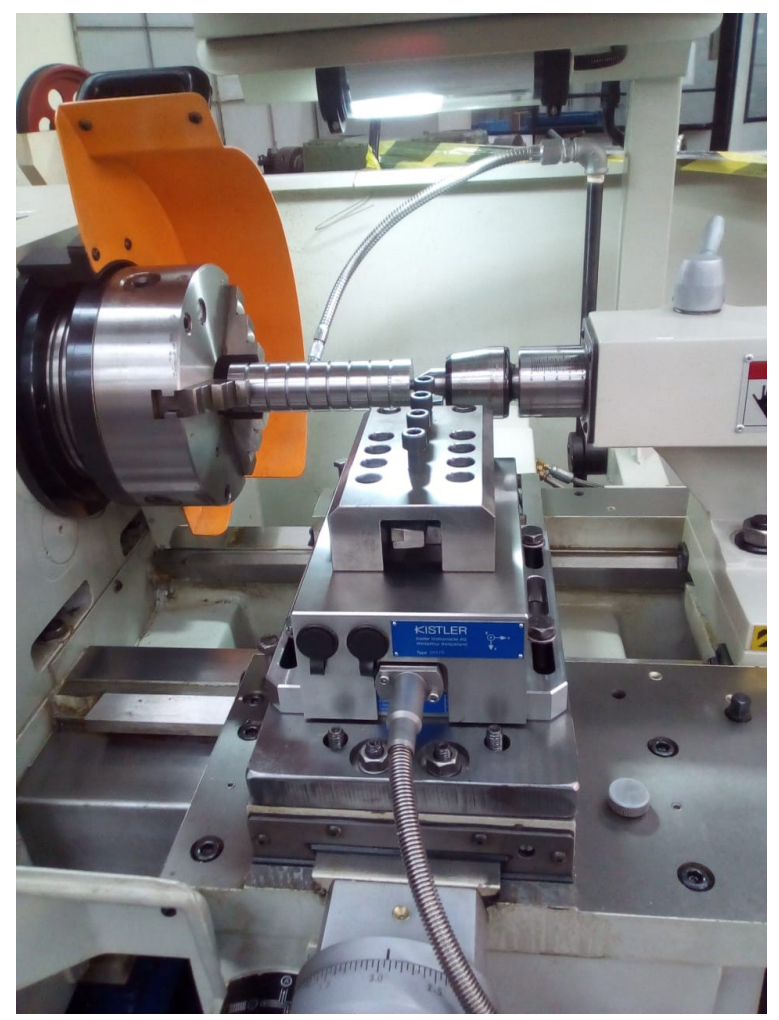

Figura 12 - Dinamometro Klister 9257B.

\subsection{Modelagem Numérica}

No presente trabalho um modelo 3D foi modelado para simular um corte ortogonal, Fig. 13. O modelo de Johnson-Cook foi usado para modelar as características térmicas, elásticas e plásticas de ambos materiais, inserto de metal duro e aço 1045. Na simulação, a peça é modelada como material dúctil e deformável e a ferramenta como um material rígido. A peça foi configurada como ENCASTRE no software ABAQUS, em outras palavras, todos os graus de liberdade são igual a zero. Não há deslocamento transversal e nem rotacional. A ferramenta foi configurada para se mover apenas na direção X, ou seja, na direção do corte. 


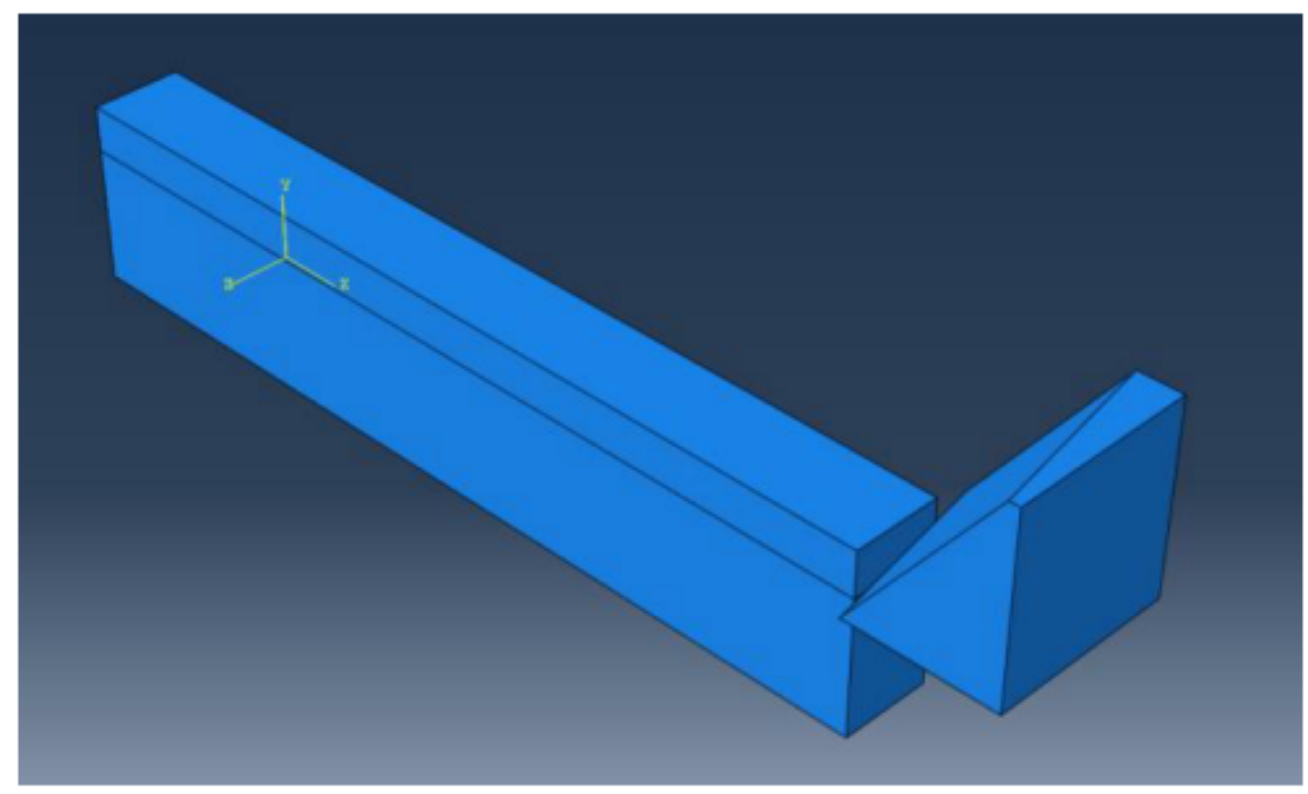

Figura 13 - Modelagem 3D da peça e ferramenta.

\subsection{Malha aptativa Lagrangina-Euleriana}

Em MEF é necessário criar uma malha efetiva para a peça. Existem duas formulações matemáticas que descrevem o deslocamento: método Lagrangeano e Euleriano. O método Lagrangeano a malha se move com o material e a Euleriana a malha permanece fixa no espaço e apenas o material que se move em relação ao nós Zetterberg (2014). O método ALE é uma combinação de ambos os métodos descritos acima. Isso significa que a malha pode fluir arbitrariamente. A malha é essencial para se obter a formação e a separação do cavaco durante a simulação. A falha proveniente do cisalhamento entre a ferramenta e o cavaco é caracterizado pelo modelo de Zorev's. Com o modelo de atrito de Zorev's atuando entre a ferramenta e o cavaco é possível prever com precisão a direção do desprendimento do cavaco durante o processo de usinagem (Gao et al., 2018). O software ABAQUS/Explicit v6.14 e o método ALE foi utilizado nessa simulação. 


\section{Resultados}

A análise das forças de corte é de extrema importância no processo de usinagem. O modelo 3D descrito previamente foi submetido a etapas de simulação numérica no software ABAQUS/Explicit. Na figura 14 e figura 15 é possível ver os elementos submetidos a forças extremas, como as tensões cisalhamento e axial. A ferramenta caminha rapidamente ao longo da peça, removendo material. Nesse momento, a força de corte se encontra em sua maior intensidade durante o processo, além de transferência de calor ao longo da peça. A combinação da deformação e fusão do material culmina na formação do cavaco, como esperado devido ao método adotado na simulação. A simulação levou 5 horas para gerar o resultado.

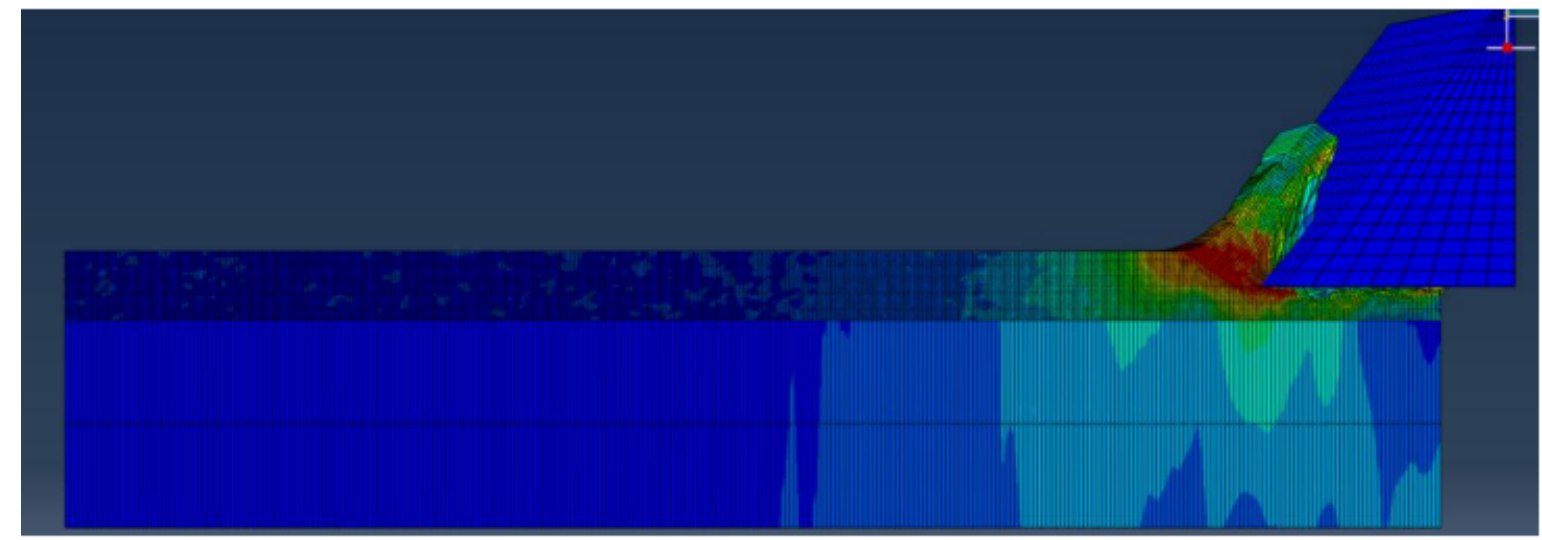

Figura 14 - Simulação de usinagem 3D.

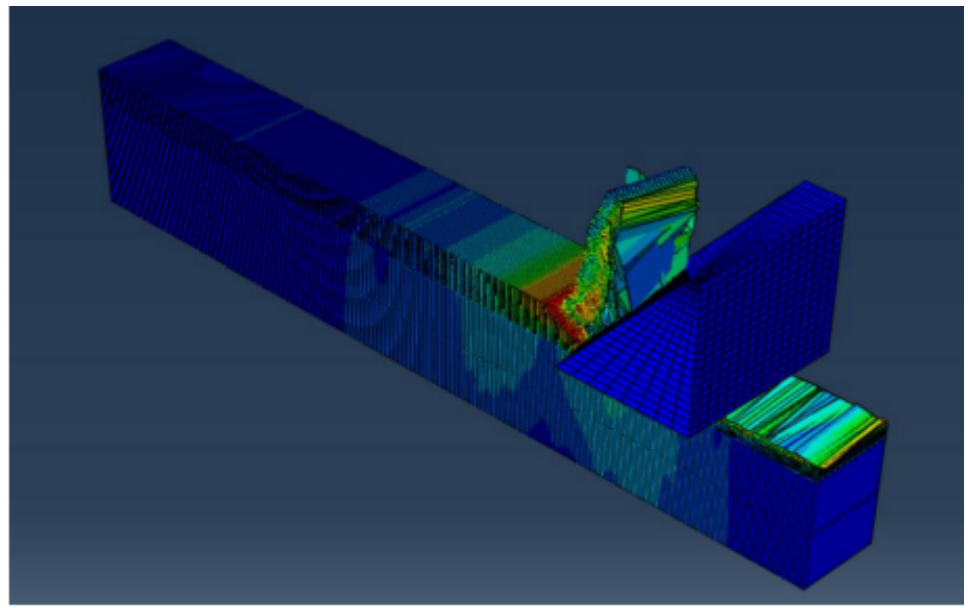

Figura 15 - Formação do Cavaco.

Como esperado, a ferramenta não sofreu deformação. Esse resultado é lógico devido ao material que compõe a peça é mais dúctil que o o material da ferramenta. Dessa forma, 
o inserto de tungstênio é ideal para usinagem do aço 1045 com os parâmetros estabelecidos neste trabalho.

Os gráficos da figura 16 compara os valores obtidos numericamente com os valores provenientes do dinamômetro estacionário Kliste. A linha contínua descreve resultados coletados pelos sensores do dinamômetro e os quadrados mostram as forças obtidas na simulação.

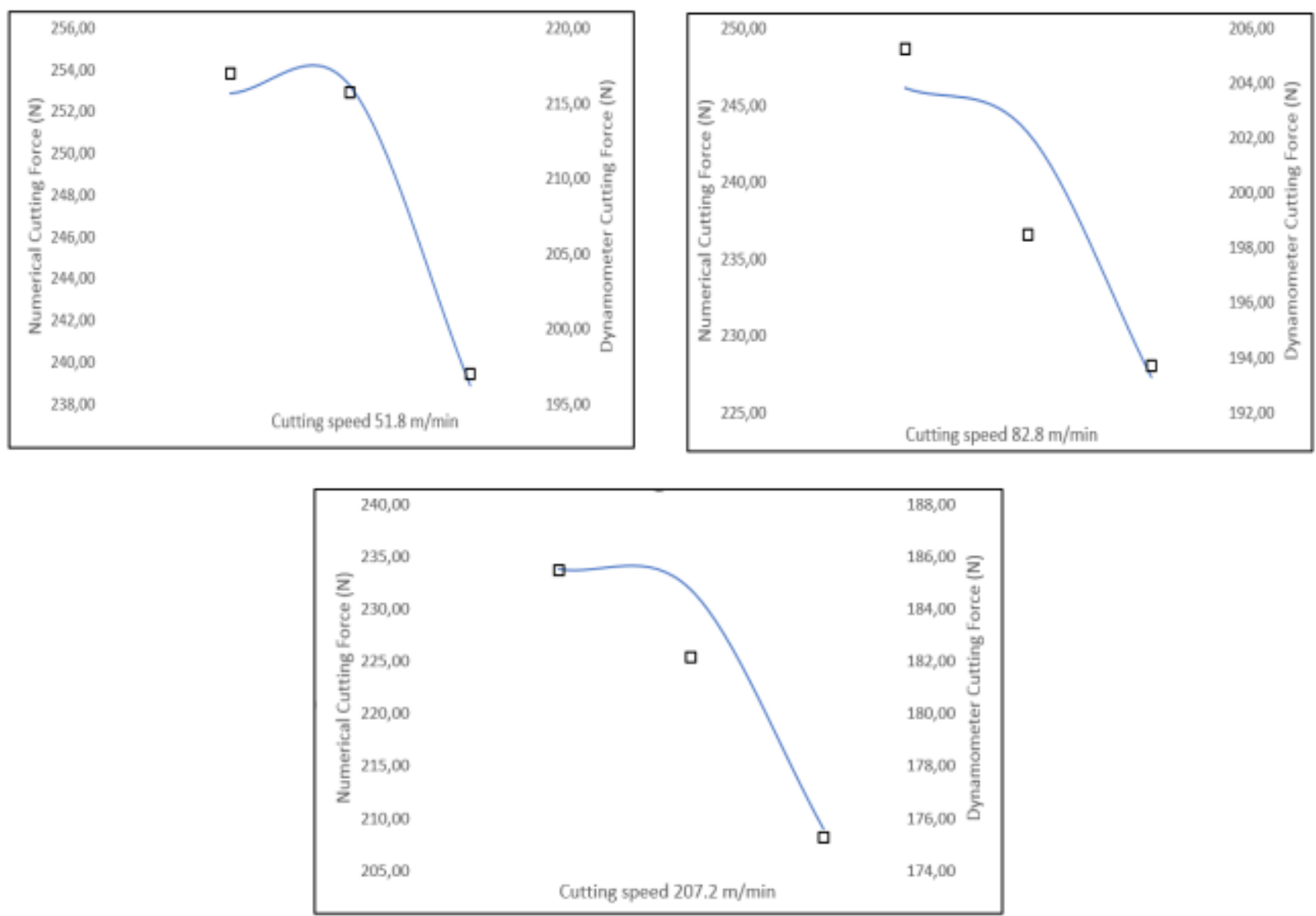

Figura 16 - Análise das forças de corte no aço 1045.

A gráficos de variação da velocidade de corte valida a teoria da usinagem de metais. A literatura diz que a força de corte tende a diminuir devido alguns fenômenos, como temperatura e alta velocidade de corte. Durante o processo de usinagem, o atrito entre a ferramenta-peça e a velocidade de corte culmina no aumento da temperatura. Por conta da alta temperatura e velocidade, o material da peça se torna ainda mais dúctil, provocando a redução da força de corte. Nos gráficos acima é possível observar esse fenômeno. O primeiro gráfico com a menor velocidade de corte tem maior força de usinagem, enquanto o último gráfico, com maior velocidade de corte, mostra uma menor força de corte. Em outras palavras, na usinagem de materiais a força de corte é totalmente relacionada com a velocidade de corte e temperatura. É possível ver a proximidade da linha 
continua dos quadrados, isso é, os valores obtidos analiticamente e numericamente são próximo. O erro está em torno de 20\%. As condições de contorno e qualidade da malha influencia diretamente nos resultados. Um modelo mais refinado pode atenuar o erro e promover a precisão dos resultados, mas o consumo de processamento computacional aumenta significativamente. 



\section{Conclusão}

Toda ferramenta possui um manual de uso desenvolvido pela fabricante. Parâmetros de uso como velocidade de corte, profundidade de corte e velocidade de avanço afetam diretamente a vida útil da ferramenta. Trabalhar com simulação é financeiramente viável. Na simulação é possível trabalhar diversas configurações de usinagem, materiais e ferramentas podem ser simuladas. Com os dados experimentais gerados na simulação é possível mostrar que a maior força de corte ocorre no primeiro contato entre ferramentapeça. Nos gráficos de força de corte é possível observar que a simulação numérica é um método atrativo e que possui resultados consistentes. Diversos fatores podem contribuir com a acurácia dos valores obtidos, tal como o refinamento da malha e as condições de contorno. Os valores coletados na simulação estão próximos aos descritos na literatura. De acordo com os artigos da área, o erro entre a simulação numérica e experimental usando o dinamômetro é próximo de $20 \%$. 



\section{Referências}

Albrecht, A., Park, S. S., Altintas, Y., and Pritschow, G. (2005). High frequency bandwidth cutting force measurement in milling using capacitance displacement sensors. International Journal of Machine Tools and Manufacture, 45(9):993-1008. Citado na página 17.

Banerjee, A., Dhar, S., Acharyya, S., Datta, D., and Nayak, N. (2015). Determination of johnson cook material and failure model constants and numerical modelling of charpy impact test of armour steel. Materials Science and Engineering: A, 640:200-209. Citado na página 31.

de Andrade Dimas, N. (2018). Modelagem numérica e experimental do corte ortogonal. Citado na página 29.

Dehkharghani, A. A. (2016). Tuning Johnson-Cook material model parameters for impact of high velocity, micron scale aluminum particles. Northeastern University. Citado na página 31.

Diniz, A., Marcondes, F., and Coppini, N. (2001). Tecnologia da usinagem dos materiais 3. ed. São Paulo, Artliber Editora. Citado 4 vezes nas páginas 11, 17, 26 e 27.

Ehmann, K., e. a. (1997). Machining process modeling: A review. Journal of Manufacturing Technology, pages 849-857. Citado na página 17.

Ferraresi, D. (1970). Usinagem dos metais, volume 1. Editôra Edgard Blücher. Citado na página 17.

Gao, Y., Ko, J. H., and Lee, H. P. (2018). 3d coupled eulerian-lagrangian finite element analysis of end milling. The International Journal of Advanced Manufacturing Technology, 98(1-4):849-857. Citado na página 36.

Gonzalo, O., e. a. (2008). Prediction of specific force coefficients from a fem cutting model. International Journal of Advanced Manufacturing Technology, pages 348-356. Citado na página 17.

Groover, M. P. (2007). Fundamentals of modern manufacturing: materials processes, and systems. John Wiley \& Sons. Citado 3 vezes nas páginas 11, 27 e 28.

Harish, A. (2019). Finite Element Method - What Is It? FEM and FEA Explained. https: //www.simscale.com/blog/2016/10/what-is-finite-element-method/, [Accessed: 15/11/2019]. Citado na página 18. 
Kalpakjian, S., Schmid, S. R., and Musa, H. (2011). Manufacturing engineering and technology: machining. China Machine Press. Citado 2 vezes nas páginas 21 e 33.

Mabrouki, T. and Rigal, J.-F. (2006). A contribution to a qualitative understanding of thermo-mechanical effects during chip formation in hard turning. Journal of Materials Processing Technology, 176(1-3):214-221. Citado na página 31.

Machado, Á. R., Coelho, R. T., Abrão, A. M., and da Silva, M. B. (2015). Teoria da usinagem dos materiais. Editora Blucher. Citado na página 21.

Machado, Á. R. and Silva, M. d. (2004). Usinagem dos metais. Departamento de Engenharia Mecânica, Universidade Federal de Uberlândia, MG, $8^{a}$ ed., 25\%p. Citado 7 vezes nas páginas 11, 17, 22, 23, 24, 25 e 28.

Nascimento, C. H. and Coelho, R. T. (2011). Estudo da distribuição de calor na região de corte durante o processo de fresamento ortogonal através do método de elelentos finitos. Citado 2 vezes nas páginas 11 e 26.

Scippa, A., e. a. (2015). Improved dynamic compensation for acurate cutting force measurements in milling applications. Mech Syst Signal Process, pages 314-324. Citado na página 17 .

Souza, R. (2003). O Método Dos Elementos Finitos Aplicado ao Problema de ConduÇão de Calor. NICAE. Citado 3 vezes nas páginas 29, 30 e 31.

Trent, E. M. and Wright, P. K. (2000). Metal cutting. Butterworth-Heinemann. Citado na página 21.

Walker, J. R. (1989). Machining fundamentals: from basic to advanced techniques. Goodheart-Willcox Company. Citado na página 17.

Wan, L., Haddag, B., Wang, D., Sheng, Y., and Yang, D. (2018). Effects of friction conditions on the formation of dead metal zone in orthogonal cutting-a finite element study. Machining Science and Technology, 22(6):934-952. Citado na página 25.

Zetterberg, M. (2014). A critical overview of machining simulations in abaqus. Citado na página 36. 